\title{
TESTING THE CLAR THEORY: CYCLIC CONJUGATION IN SOME BENZO- AND NAPHTHALENO-ANNELATED OVALENES
}

\author{
Ivan Gutman, ${ }^{1}$ Jelena Đurđević, ${ }^{1}$ Sabina Gojak-Salimovićc ${ }^{2}$ \\ ${ }^{1}$ Faculty of Science, University of Kragujevac, P.O. Box 60, 34000 Kragujevac, Serbia \\ E-mail: gutman@kg.ac.rs, jddjurdjevic@gmail.com \\ ${ }^{2}$ Department of Chemistry, Faculty of Science, University of Sarajevo, \\ 71000 Sarajevo, Bosnia and Herzegovina \\ E-mail:sgojak@pmf.unsa.ba
}

(Received December 6, 2013)

\begin{abstract}
Cyclic conjugation (assessed by its energy effect) is studied in benzo- and naphthaleno-annelated ovalenes, in which annelation is in positions $d$ and $o$. These benzenoid species possess a large number of Clar aromatic sextet formulas, making the application of Clar theory difficult and ambiguous. Some predictions based on Clar formulas are not in agreement with the calculated energy effects. This indicates that Clar theory is not universally applicable, even in the case of fully conjugated benzenoid molecules.
\end{abstract}

\section{Introduction}

Benzenoid hydrocarbons form a class of conjugated $\pi$-electron systems whose theory is elaborated in due detail [1-7]. Cyclic conjugation in benzenoid hydrocarbons was especially much studied, see the papers [8-14] and the references cited therein. Erich Clar's "aromatic sextet theory" [1,3] provides the simplest and most direct way by which one can get an insight into the dominant modes of cyclic conjugation of a benzenoid molecule. In Clar theory, so-called "Clar formulas" are constructed by placing "aromatic sextets" into some rings of a benzenoid molecule, obeying certain formal rules [3,15] (cf. Figs. 1-3). Rings in which "aromatic sextets" are located are predicted to have a high intensity of cyclic conjugation. The original version of Clar's method is qualitative and has no direct foundation in quantum theory. Eventually, much effort has been done to provide a quantitative and theoretically founded re-formulation of Clar's model (see the recent works [16-24] and the references cited therein). In our earlier studies [13,14,24-27], examples of benzenoid hydrocarbons were found in which the predictions of Clar 
theory were violated. However, the benzenoid systems in these examples contained fixed single and double carbon-carbon bonds, and thus it could be argued that Clar theory was not strictly applicable to them. We have now detected a class of fully conjugated benzenoid molecules (i.e., molecules without fixed single and double carbon-carbons bonds), in which the modes of cyclic conjugation are not in full agreement with the Clar model. These are the benzo- and naphthaleno-derivatives of ovalene, in which annelation is in position $d$ and $o$, see Fig. 1.

A quantitative measure of cyclic conjugation in $\pi$-electron systems, especially in benzenoid hydrocarbons, is achieved by the energy-effect of the respective cycles. Details of this method have been earlier described several times, e.g., in the articles [8-10] and in the review [11]. Thus, $e f=e f(Z)$ is the energy-effect caused by cyclic conjugation of $\pi$-electrons along the cycle $Z$ in the underlying conjugated molecule. As usual, in what follows, ef will be expressed in the units of the carbon-carbon resonance integral $\beta$, where $\beta \approx-137 \mathrm{~kJ} \mathrm{~mol}^{-1}$. Note that since $\beta$ is negative-valued, large (positive) ef-values mean a large stabilizing energy-effect caused by conjugation in the cycle $Z$, providing an energetic measure of the intensity of cyclic conjugation in $Z$.

The $e f$-values of all six-membered rings of ovalene (1) and its annelated congeners (2-6, see Fig. 1) are collected in Table 1.
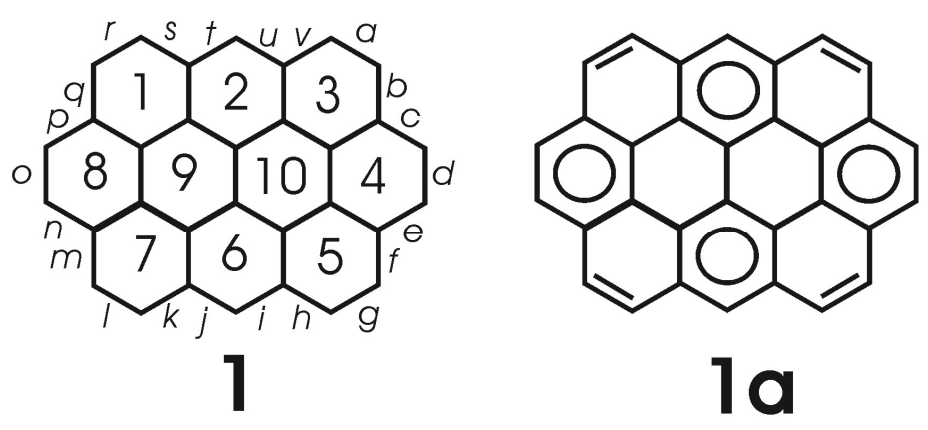

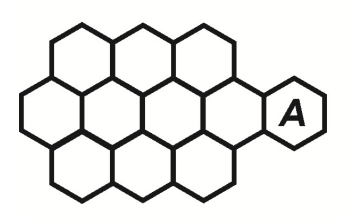

2

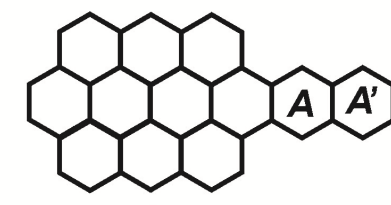

3

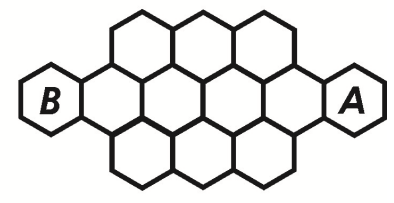

4

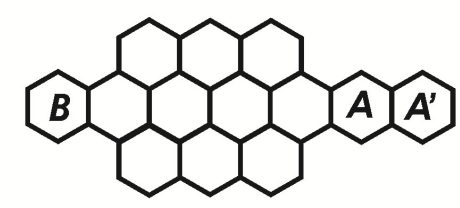

5

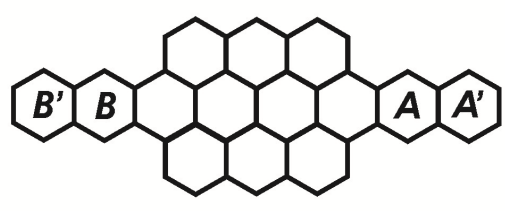

6

Fig. 1 - Ovalene (1), its unique Clar formula (1a), its benzo- and naphthaleno-annelated derivatives studied in this work, and the labelling of their rings. 


\begin{tabular}{|c|c|c|c|c|c|c|c|c|c|c|c|}
\hline comp. & $\mathbf{1}$ & $\mathbf{2}$ & $\mathbf{3}$ & $\mathbf{4}$ & $\mathbf{8}$ & $\mathbf{9}$ & $\mathbf{1 0}$ & $\mathbf{A}$ & $\mathbf{A}^{\prime}$ & $\mathbf{B}$ & $\mathbf{B}^{\prime}$ \\
\hline $\mathbf{1}$ & 0.0567 & 0.0575 & 0.0567 & 0.0760 & 0.0760 & 0.0336 & 0.0336 & & & & \\
\hline $\mathbf{2}$ & 0.0549 & 0.0562 & 0.0699 & 0.0344 & 0.0771 & 0.0345 & 0.0309 & 0.1588 & & & \\
\hline $\mathbf{3}$ & 0.0546 & 0.0557 & 0.0738 & 0.0276 & 0.0775 & 0.0348 & 0.0308 & 0.0801 & 0.1052 & & \\
\hline $\mathbf{4}$ & 0.0676 & 0.0551 & 0.0676 & 0.0350 & 0.0350 & 0.0316 & 0.0316 & 0.1567 & & 0,1567 & \\
\hline $\mathbf{5}$ & 0.0670 & 0.0547 & 0.0713 & 0.0280 & 0.0352 & 0.0318 & 0.0315 & 0.0794 & 0.1047 & 0.1561 & \\
\hline $\mathbf{6}$ & 0.0706 & 0.0544 & 0.0706 & 0.0281 & 0.0281 & 0.0317 & 0.0317 & 0.0791 & 0.1046 & 0.0791 & 0.1046 \\
\hline
\end{tabular}

Table 1 - Energy effects (in $\beta$-units, $\beta \approx-137 \mathrm{~kJ} \mathrm{~mol}^{-1}$ ) of the rings of ovalene (1) and its annelated derivatives 2-6, see Fig. 1. The labeling of the rings is also indicated in Fig. 1.

The energy-effects of the rings 5, 6 , and 7 are omitted because, by symmetry, these are always equal to the $e f$-values of the rings 3,2 , and 1 , respectively.

\section{Agreements with Clar theory: Ovalene, benzo[d]ovalene, naphthaleno[d]ovalene}

Ovalene has a unique Clar formula, depicted in Fig. 1. According to it, the most intense cyclic conjugation is to be expected in the rings $2,4,6$, and 8 . Indeed, $e f(2)=e f(6)=0.0575$ is somewhat greater than $e f(1)=e f(3)=e f(5)=e f(7)=0.0567$, although the difference is slight. As expected, the rings 4 and 8 have the greatest, whereas the "empty" rings 9 and 10 the smallest $e f$ value.

The situation with benzo $[d]$ ovalene and naphthaleno $[d]$ ovalene is much less simple.

Namely, benzo $[d]$ ovalene (2) has 6, whereas naphthaleno[d]ovalene (3) has 11 Clar formulas. The Clar formulas of benzo[d]ovalene are shown in Fig. 2.
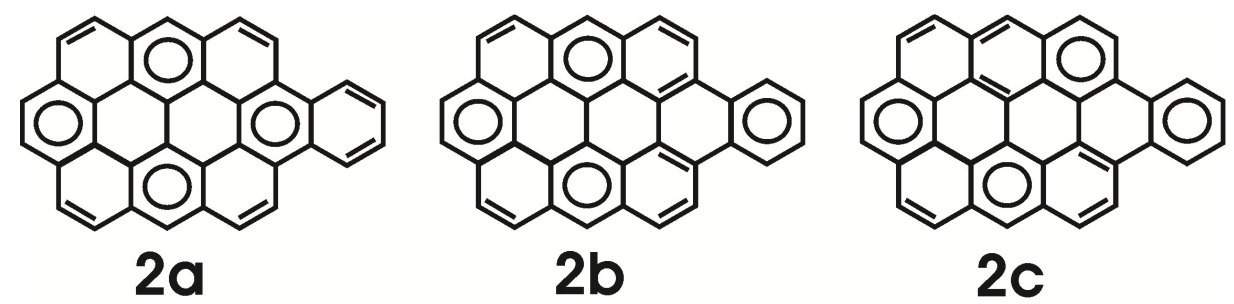

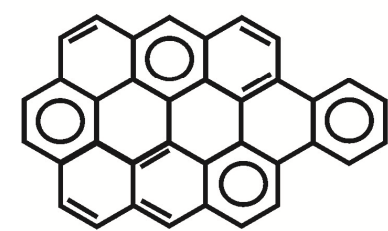

2d

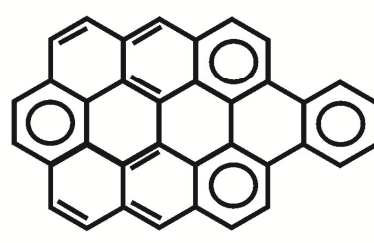

$2 e$

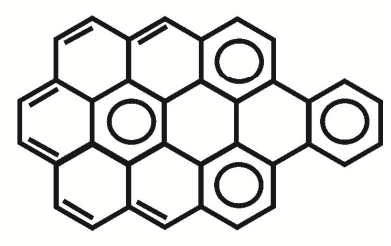

$2 f$

Fig. 2 - The six Clar aromatic sextet formulas of benzo[ $d]$ ovalene. In formula $2 \mathrm{f}$ an aromatic sextet is located in ring 9, whereas no one of these formulas has an aromatic sextet in ring 10. This fact is in good agreement with the increased $e f$-value of ring 9 and decreased $e f$-value of ring 10 , relative to ovalene (cf. 
Already the fact that $\mathbf{2}$ and $\mathbf{3}$ have so many different aromatic sextet formulas, implies that the application of the Clar model to these benzenoid hydrocarbons is neither simple nor unambiguous. The assumption [18,19,21] that all Clar formulas are equally important is evidently an oversimplification. For instance, according to the ef-method, cyclic conjugation in the ring $\mathrm{A}$ of $\mathbf{2}$ is almost five times stronger than in the ring 4. Therefore, the Clar formula $\mathbf{2 a}$

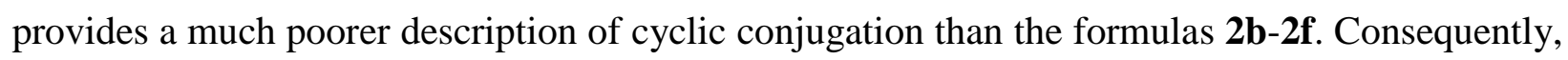
the weight by which formula $\mathbf{2 a}$ is taken into consideration should be much smaller than the weight of the remaining five Clar formulas. However, no matter how we choose such a "weight", one Clar formula, namely $\mathbf{2 f}$, implies an increased intensity of cyclic conjugation in the ring 9, but not in the ring 10. In harmony with this qualitative prediction is $e f(9)=0.0345$ and $e f(10)=$ 0.0309, which should be compared with $e f(9)=e f(10)=0.0336$ for ovalene (1).

An identical conclusion holds also for naphthaleno $[d]$ ovanele $(3)$, for which $e f(9)=$ 0.0348 and $e f(10)=0.0308$.

\section{Disagreements with Clar theory: $[d, o]$-diannelated ovalenes}

The ovalene derivatives annelated in both positions $d$ and $o$, namely the compounds $\mathbf{4 , 5}$, and 6 (cf. Fig. 1), possess a remarkably large number of Clar formulas: 20 for $\mathbf{4}, 34$ for $\mathbf{5}$, and 57 for 6. Lacking a procedure for ranking these formulas by relevance, it is hard to give any sound

prediction (based on Clar theory) for the modes of cyclic conjugation in $\mathbf{4 , 5}$, and $\mathbf{6}$. Yet, one conclusion is doubtless. All the three benzenoid systems 4, 5, and $\mathbf{6}$ have Clar formulas in which an aromatic sextet is located in the rings 9 or 10 . These formulas are depicted in Fig. 3. No matter what is the relevance of these formulas relative to other Clar formulas, they imply that the magnitude of cyclic conjugation in the rings 9 and 10 should be greater than in the respective rings of the non-annelated ovalene (1). In all the three benzenoid systems $\mathbf{4}, \mathbf{5}$, and $\mathbf{6}$, the efvalues of the rings 9 and 10 are between 0.0315 and 0.0318 (see Table 1). On the other hand, in ovalene, $e f(9)=e f(10)=0.0336$, which contradicts the prediction of Clar theory. 

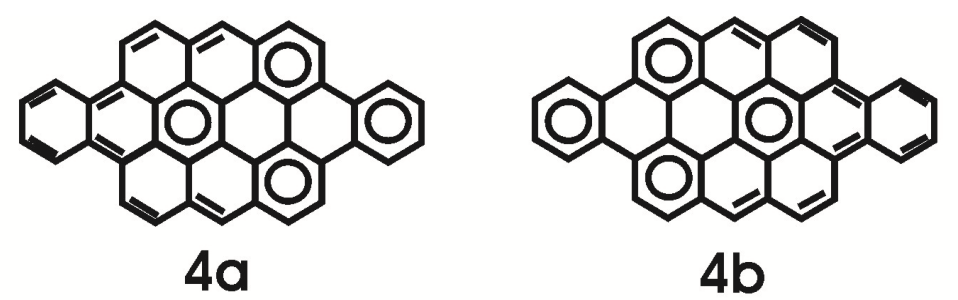

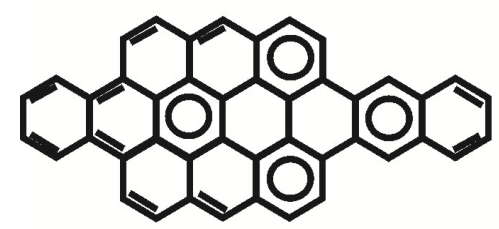

$5 a$

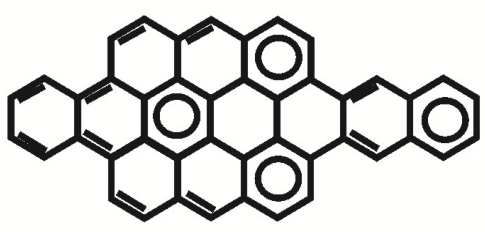

$5 b$

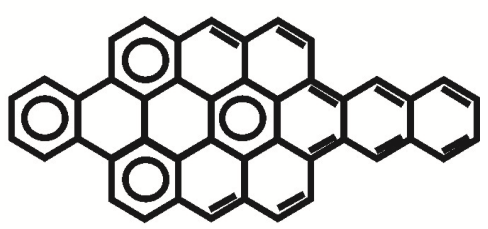

$5 \mathrm{c}$
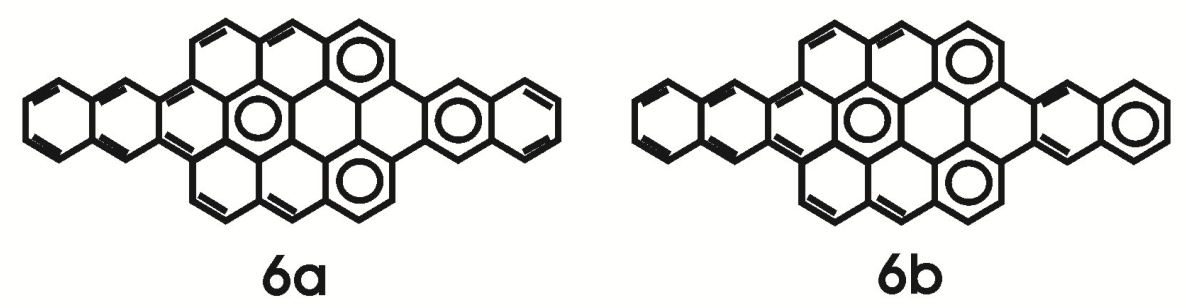

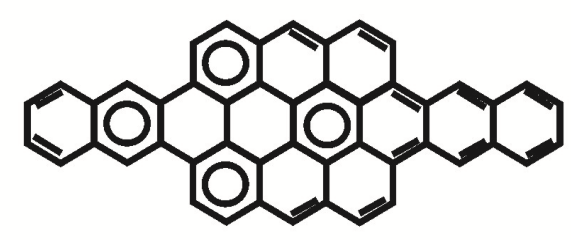

$6 c$

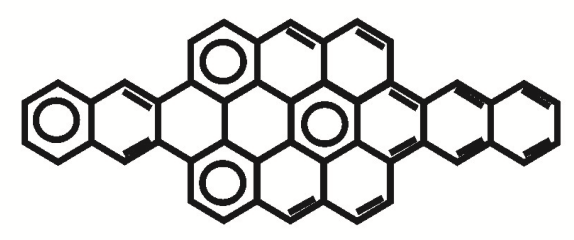

6d

Fig. $3 \mathbf{- 4 a}$ and $\mathbf{4 b}$ are two of the 20 Clar formulas of dibenzo[d,o]ovalene (4); $\mathbf{5 a}, \mathbf{5 b}$, and $\mathbf{5 c}$ are three of the 34 Clar formulas of benzo[o $[o$ naphthaleno $[d]$ ovalene $(\mathbf{5}) ; \mathbf{6 a}, \mathbf{6 b}, \mathbf{6 c}$, and $\mathbf{6 d}$ are four of the 57 Clar formulas of dinaphthaleno[ $d, o]$ ovalene (6). These are the Clar formulas in which an aromatic sextet is located in the rings 9 or 10, implying that the magnitude of cyclic conjugation in these rings is greater than in ovalene (1). This prediction of Clar theory is erroneous (cf. Table 1).

\section{Discussion and concluding remarks}

Several examples documenting disagreements between the actual $\pi$-electron structure of benzenoid hydrocarbons, and what is predicted on the basis of the Clar aromatic sextet theory, have been reported in the past [13,14,24-27]. The cases studied in this work offer a further indication of such disagreements. Clar theory is not quantitative in nature, and therefore it is not easy to recognize it possible flaws. The study of cyclic conjugation in the central rings 9 and 10 provides an example of a qualitative disagreement: instead of an increase of the magnitude of cyclic conjugation (as forecasted by Clar theory) we encounter a decrease. 
The effect observed and discussed in this note is relatively weak, around 5\%. We expect that much more pronounced violations of Clar- and Kekulé-structure based theories would be found at the benzo- and naphthaleno-annelated derivatives of the higher members of the coronene/ovalene homologous series. Research along these lines is intended to be done in the future.

\section{REFERENCES}

[1] E. Clar, The Aromatic Sextet, Wiley, London, 1972.

[2] J. R. Dias, Handbook of Polycyclic Hydrocarbons. Part A. Benzenoid Hydrocarbons, Elsevier, Amsterdam, 1987.

[3] I. Gutman, S. J. Cyvin, Introduction to the Theory of Benzenoid Hydrocarbons, Springer, Berlin, 1989.

[4] I. Gutman, S. J. Cyvin (Eds.), Advances in the Theory of Benzenoid Hydrocarbons, Springer, Berlin, 1990.

[5] I. Gutman (Ed.), Advances in the Theory of Benzenoid Hydrocarbons II, Springer, Berlin, 1992.

[6] M. Zander, Polycyclische Aromaten, Teubner, Stuttgart, 1995.

[7] J. R. Dias, Mathematics of periodic tables of benzenoid hydrocarbons, J. Chem. Inf. Model. 47 (2007) 707-715.

[8] B. Furtula, I. Gutman, N. Turković, Relation between electron and energy contents of hexagons in catacondensed benzenoid hydrocarbons, Indian J. Chem. 44A (2005) 9-12.

[9] I. Gutman, S. Milosavljević, B. Furtula, N. Cmiljanović, Relation between electron and energy contents of hexagons in pericondensed benzenoid hydrocarbons, Indian J. Chem. 44A (2005) 13-17.

[10] I. Gutman, S. Stanković, R. Kovačević, J. Đurđević, B. Furtula, Anomalous cyclic conjugation in benzenoid molecules with a small number of Kekulé structures, Indian J. Chem. 44A (2005) 1751-1755.

[11] I. Gutman, Cyclic conjugation energy effects in polycyclic $\pi$-electron systems, Monatsh. Chem. 136 (2005) 1055-1069.

[12] A. T. Balaban, J. Đurđević, I. Gutman, S. Jeremić, S. Radenković, Correlations between local aromaticity indices of bipartite conjugated hydrocarbons, J. Phys. Chem. A 114 (2010) 5870-5877. 
[13] I. Gutman, J. Đurđević, Z. Matović, M. Marković, Verifying the modes of cyclic conjugation in tetrabenzo[bc,ef,op,rs]circumanthracene, J. Serb. Chem. Soc. 77 (2012) 1401-1408.

[14] I. Gutman, J. Đurđević, S. Radenković, Z. Matović, Anomalous cyclic conjugation in the perylene/bisanthrene homologous series, Monatsh. Chem. 143 (2012) 1649-1653.

[15] A. T. Balaban, Clar formulas: How to draw and how not to draw formulas of polyciclic aromatic hydrocarbons, Polyc. Arom. Comp. 24 (2004) 83-89.

[16] J. Aihara, $\pi$-Electron currents induced in polycyclic benzenoid hydrocarbons and their relationship to Clar structures, J. Phys. Chem. A 107 (2003) 11553-11557.

[17] G. Portella, J. Poater, M. Solá, Assessment of Clar's aromatic $\pi$-sextet rule by means of PDI, NICS and HOMA indicators of local aromaticity, J. Phys. Org. Chem. 18 (2005) $785-$ 791.

[18] M. Randić, A. T. Balaban, Partitioning of $\pi$-electrons in rings for Clar structures of benzenoid hydrocarbons, J. Chem. Inf. Model, 46 (2006) 57-64.

[19] M. Randić, A. T. Balaban, $\pi$-Electron partitions, signatures, and Clar structures of selected benzenoid hydrocarbons, J. Phys. Chem. A 112 (2008) 4148-4157.

[20] K. Salem, S. Klavžar, A.Vesel, P. Žigert, The Clar formulas of a benzenoid system and the resonance graph, Discr. Appl. Math. 157 (2009) 2565-2569.

[21] M. Randić, D. Plavšić, Algebraic Clar formulas - Numerical representation of Clar structural formula, Acta Chim. Sloven. 58 (2011) 448-457.

[22] C. P. Chou, H. Witek, An algorithm and FORTRAN program for automatic computation of the Zhang-Zhang polynomial of benzenoids, MATCH Commun. Math. Comput. Chem. 68 (2012) 3-30.

[23] C. P. Chou, Y. Li, H. A. Witek, Zhang-Zhang polynomials of various classes of benzenoid systems, MATCH Commun. Math. Comput. Chem. 68 (2012) 31-64.

[24] I. Gutman, S. Radenković, M. Antić, J. Đurđević, A test of Clar aromatic sextet theory, J. Serb. Chem. Soc. 78 (2013) 1539-1546.

[25] I. Gutman, N. Turković, J. Jovičić, Cyclic conjugation in benzo-annelated perylenes: How empty is the "empty" ring?, Monatsh. Chem. 135 (2004) 1389-1394.

[26] I. Gutman, N. Turković, B. Furtula, On distribution of $\pi$-electrons in rhombus-shaped benzenoid hydrocarbons, Indian J. Chem. 45A (2006) 1601-1604.

[27] S. Gojak-Salimović, I. Gutman, J. Đurđević, M. Janošević, Cyclic conjugation in benzoannelated ovalenes, Bull. Chem. Technol. Bosn. Herz. 40 (2013) 17-20. 